\title{
$m$-submultisets and $m$-permutations of multisets elements
}

\author{
Makhnei O.V. ${ }^{凶}$, Pylypiv V.M., Zatorskii R.A.
}

\begin{abstract}
The article is devoted to two classical combinatorial problems on multisets, which in the existing literature are given unjustifiably little space. Namely, the calculation of the number of all submultisets of power $m$ for an arbitrary multiset and the number of $m$-permutations of such multisets. The first problem is closely related to the width of a partially ordered set of all submultisets of a multiset with the inclusion $\subseteq$. The article contains some important classes of multisets. Combinatorial proofs of problems on the number of $m$-submultisets and $m$-permutations of multiset elements are considered. In the article, on the basis of the generatrix method, thrifty algorithms for calculating $m$-submultisets and $m$-permutations of multiset elements are constructed. The paper also provides a brief overview of the results that are related to this area of research.
\end{abstract}

Key words and phrases: multiset, permutation.

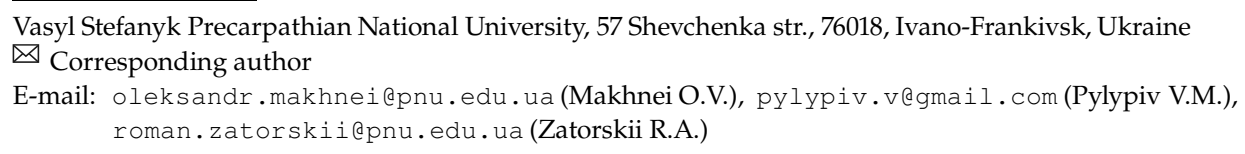

\section{Introduction}

In discrete mathematics, problems of investigating sets of objects with identical objects often arise. Therefore, from the middle of the last century the concept of multiset (see $[1,14,15])$ begins to gain more and more value. Since the multiset is a natural generalization of the set, the problems of generalization of the classical results of combinatorics of finite sets naturally arise. Thus, in the paper [7], C. Green, D.J. Kleitman, in fact, consider the problem of calculating the number of $m$-submultisets of a multiset. However, in the general case, just a few problems were solved. Usually, authors are limited to considering only some partial but very important classes of multisets.

In the papers [3,5], P. Cartier, D. Foata introduced the concept of "intercalation product" $\alpha \top \beta$, which extended a number of known results concerning ordinary permutations of sets to the case of multisets. In the book [10], D. Knuth develops combinatorial techniques for multisets. Using the theorem that each permutation of a multiset can be written as

$$
\sigma_{1} \top \sigma_{2} \top \ldots \top \sigma_{t}, \quad t \geqslant 0,
$$

where $\sigma_{j}$ are cycles such that their elements are not repeated, D. Knuth gives examples of enumeration of permutations of multisets with some restrictions. The case $t=0$ corresponds to the division of the empty multiset into cycles which is carried out using the intercalation product of Foata. This can be found in more detail in [10].

The paper [13] is very useful for applications. 
The so-called nondecreasing series in the permutations of multisets (see [2,4]) have important applications in the study of "order statistics". In the case of a constant multiset $\left\{1^{p}, 2^{p}, \ldots, m^{p}\right\}$ in the paper [11, pp. 212-213], P.A. MacMahon showed that the number of permutations with $k+1$ series is equal to the number of permutations with $m p-p-k+1$ series. Also, by the generatrix method, P.A. MacMahon proved that the number of permutations of the multiset $\left\{1^{n_{1}}, 2^{n_{2}}, \ldots, m^{n_{m}}\right\}$ with $k$ series is equal to

$$
\sum_{j=0}^{k}(-1)^{j}\left(\begin{array}{c}
n+1 \\
j
\end{array}\right)\left(\begin{array}{c}
n_{1}-1+k-j \\
n_{1}
\end{array}\right)\left(\begin{array}{c}
n_{2}-1+k-j \\
n_{2}
\end{array}\right) \ldots\left(\begin{array}{c}
n_{m}-1+k-j \\
n_{m}
\end{array}\right),
$$

where $n=n_{1}+n_{2}+\ldots+n_{m}$.

An interesting approach for enumerating submultisets of multisets is proposed in [9].

Sometimes a continual apparatus is used to solve discrete mathematics problems. For instance, in [6], using the generatrix method, V.V. Gotsulenko proved an integral formula for calculating the number of $m$-submultisets of the given multiset

$$
\left|C^{m}(A)\right|=\frac{1}{2 \pi} \int_{-\pi}^{\pi} \exp (-i m \varphi) \prod_{j=1}^{n} \frac{\exp \left\{i\left(k_{j}+1\right) \varphi\right\}-1}{\exp \{i \varphi\}-1} d \varphi,
$$

where $i=\sqrt{-1}$. In [6], the problem for $m$-submultisets of a multiset is also somewhat generalized.

Note also that the principle of inclusion-exclusion can be used to find the number of submultisets of a multiset. In the case of constant multisets, it gives the following result of O.G. Ganyushkin:

$$
C_{m}(A)=\sum_{i \geqslant 0}(-1)^{i}\left(\begin{array}{c}
n \\
i
\end{array}\right)\left(\begin{array}{c}
n+m-1-i(q+1) \\
n-1
\end{array}\right) .
$$

However, in the general case, this approach leads to an exponential algorithm.

This paper contains some important classes of multisets. Combinatorial proofs of problems on the number of $m$-submultisets and $m$-permutations of multiset elements are considered (see Theorems 1 and 9). Note that these theorems give exponential algorithms for finding the corresponding numbers.

The most economical algorithms for calculating $m$-submultisets and $m$-permutations of multiset elements are algorithms that are constructed using the generatrix method. They are given at the end of this work (see Algorithms 1 and 2).

This paper is a survey and contains part earlier published results in Ukrainian and Russian in the works [16-19]. Due to their inaccessibility to the English-speaking reader, these results are included in this publication.

\section{Auxiliary concepts}

The multiset $A$ means an arbitrary disordered set of elements of some set $[A]$, which we call the base of this multiset. Therefore, an arbitrary multiset can be written in the canonical form

$$
A=\left\{a_{1}^{k_{1}}, a_{2}^{k_{2}}, \ldots, a_{n}^{k_{n}}\right\}
$$

where $[A]=\left\{a_{1}, a_{2}, \ldots, a_{n}\right\}$ and indices $k_{i}$ of elements $a_{i}$ indicate the multiplicity of occurrence of the element $a_{i}$ to the multiset $A$. We can assume without loss of generality that

$$
k_{1} \geqslant k_{2} \geqslant \ldots \geqslant k_{n} \text {. }
$$


The multiset $A^{\prime}=\left\{k_{1}, k_{2}, \ldots, k_{n}\right\}$ of indices of the multiset (1) is called its primary specification. Suppose the primary specification $A^{\prime}$ of the multiset (1) is represented in the canonical form

$$
A^{\prime}=\left\{1^{\lambda_{1}}, 2^{\lambda_{2}}, \ldots, r^{\lambda_{r}}\right\}
$$

where

$$
r=\max \left(k_{1}, k_{2}, \ldots, k_{n}\right)
$$

then the multiset of its indices $A^{\prime \prime}=\left\{\lambda_{1}, \lambda_{2}, \ldots, \lambda_{r}\right\}$ is called the secondary specification of the multiset $A$.

If $i$ does not belong to the multiset $A^{\prime}$, then we assume that $\lambda_{i}=0$. Note that for the secondary specification of the multiset (1) we have the equality

$$
|A|=\lambda_{1}+2 \lambda_{2}+\ldots+r \lambda_{r}
$$
if

Multisets $A=\left\{a_{1}^{k_{1}}, a_{2}^{k_{2}}, \ldots, a_{n}^{k_{n}}\right\}$ and $\bar{A}=\left\{a_{1}^{\overline{k_{1}}}, a_{2}^{\overline{k_{2}}}, \ldots, a_{r}^{\overline{k_{r}}}\right\}$ are called the adjoint multisets

$$
\overline{k_{i}}=\left|\left\{k_{j}: k_{j} \geqslant i\right\}\right|=\left|\left\{j: k_{j} \geqslant i\right\}\right|, \quad i=1,2, \ldots, r, \quad j=1,2, \ldots, n .
$$

Here $r$ is given by the formula (2).

Let us remark that $\overline{k_{i}}$ has a certain combinatorial meaning. Namely, $\overline{k_{i}}$ is the maximum number of groups of $i$ identical elements that can be chosen from the multiset (1).

If the equality $\bar{A}=A$ holds true, then the multiset $A$ is called the multiset with a selfadjoint primary specification or the self-adjoint multiset.

If $A$ and $\bar{A}$ are the adjoint multisets and

$$
\begin{gathered}
A^{\prime}=\left\{k_{1}, \ldots, k_{n}\right\}, \quad A^{\prime \prime}=\left\{\lambda_{1}, \ldots, \lambda_{r}\right\}, \quad \bar{A}^{\prime}=\left\{\overline{k_{1}}, \ldots, \overline{k_{r}}\right\}, \\
\bar{A}^{\prime \prime}=\left\{\overline{\lambda_{1}}, \ldots, \overline{\lambda_{n}}\right\}, \quad r=\max \left(k_{1}, k_{2}, \ldots, k_{n}\right),
\end{gathered}
$$

then between the elements of their specifications, in addition to relationship (4), the following 11 relationships hold

$$
\begin{gathered}
k_{i}=\left|\left\{\lambda_{j}+\ldots+\lambda_{r}: \lambda_{j}+\ldots+\lambda_{r} \geqslant i\right\}\right|, \quad i=1, \ldots, n, \quad j=1, \ldots, r, \\
\overline{\lambda_{i}}=\left|\left\{j: \overline{k_{j}}=i\right\}\right|, \quad i=1, \ldots, n, \quad j=1, \ldots, r, \\
\overline{k_{i}}=\left|\left\{\overline{\lambda_{j}}+\ldots+\overline{\lambda_{r}}: \overline{\lambda_{j}}+\ldots+\overline{\lambda_{r}} \geqslant i\right\}\right|, \quad i=1, \ldots, r, \quad j=1, \ldots, n, \\
\lambda_{i}=\left|\left\{j: k_{j}=i\right\}\right|, \quad i=1, \ldots, r, \quad j=1, \ldots, n, \\
k_{i}=\left|\left\{\overline{k_{j}}: \overline{k_{j}} \geqslant i\right\}\right|, \quad i=1, \ldots, n, \quad j=1, \ldots, r, \\
\overline{\lambda_{i}}=\left|\left\{j: \lambda_{j}+\ldots+\lambda_{r}=i\right\}\right|, \quad i=1, \ldots, n, \quad j=1, \ldots, r, \\
\lambda_{i}=\left|\left\{j: \overline{\lambda_{j}}+\ldots+\overline{\lambda_{r}}=i\right\}\right|, \quad i=1, \ldots, r, \quad j=1, \ldots, n, \\
M \bar{\lambda}=k, \\
M^{-1} k=\bar{\lambda}, \\
M \lambda=\bar{k}, \\
M^{-1} \bar{k}=\lambda .
\end{gathered}
$$

In equalities (12) and (13), $k$ and $\bar{\lambda}$ are $n$-dimensional column vectors such that their coordinates coincide with the elements of the specifications $k(A)$ and $k^{2}(\bar{A})$ accordingly. In equalities 
(12) and (13), $M$ and $M^{-1}$ are square matrices of order $n$ of the next form

$$
M=\left(\begin{array}{ccccc}
1 & 1 & \cdots & 1 & 1 \\
0 & 1 & \cdots & 1 & 1 \\
\cdots & \ldots & \cdots & \ldots & \ldots \\
0 & 0 & \cdots & 1 & 1 \\
0 & 0 & \cdots & 0 & 1
\end{array}\right), \quad M^{-1}=\left(\begin{array}{ccccc}
1 & -1 & \cdots & 0 & 0 \\
0 & 1 & \cdots & 0 & 0 \\
\cdots & \ldots & \cdots & \cdots & \cdots \\
0 & 0 & \cdots & 1 & -1 \\
0 & 0 & \cdots & 0 & 1
\end{array}\right) .
$$

In equalities (14) and (15), $\lambda$ and $\bar{k}$ are similar $r$-dimensional column vectors, $M$ and $M^{-1}$ are similar matrices of order $r$.

Remark 1. Since $k(\overline{\bar{A}})=k(A)$, it follows that formulas (4), (5), (6), (10), (12), (13) are analogous to formulas (7), (8), (9), (11), (14), (15) correspondingly. In fact, formulas (12), (13) establish the one-to-one correspondence between the sets of solutions of equation (3) and the equation $|A|=\overline{\lambda_{1}}+2 \overline{\lambda_{2}}+\ldots+n \overline{\lambda_{n}}$, which is analogous to equation (3). A similar conclusion can be made for formulas (14), (15).

Finally, we give a well-known statement about a cardinality of multiboolean of the multiset (1).

Proposition 1. If $A=\left\{a_{1}^{k_{1}}, a_{2}^{k_{2}}, \ldots, a_{n}^{k_{n}}\right\}$ and $C(A)$ is a set of all submultisets of the multiset $A$, then

$$
|C(A)|=\prod_{i=1}^{n}\left(k_{i}+1\right)
$$

\section{Some classes of multisets and their specifications}

1. The multiset with a positive integer function of a natural argument is the multiset of the form

$$
A=\left\{a_{1}^{g(1)}, a_{2}^{g(2)}, \ldots, a_{n}^{g(n)}\right\},
$$

where $g: \mathbb{N} \rightarrow \mathbb{N}$ is some nondecreasing function that satisfies the inequality $g(i) \geqslant i$ for all $i \in \mathbb{N}$.

2. The multiset with a continuous function $f$ is the multiset of the form

$$
A=\{[f(1)],[f(2)], \ldots,[f(n)]\},
$$

where $f$ is some continuous increasing function

$$
f: D \rightarrow E, \quad D=[1, n], \quad E \supseteq[1,[f(n)]],
$$

that satisfies the inequality $f(x) \geqslant x,[\cdot]$ is the integer part of a number. Specification (17) is a partial case of the multiset (16).

For example, for the function $f(x)=\exp (x)$ and $n=5$ the first derivative of the multiset has the form

$$
A^{\prime}=\{2,7,20,54,148\} \text {. }
$$

3. The linear multiset is the multiset that has the form

$$
A=\left\{a_{1}^{p+q}, a_{2}^{p+2 q}, \ldots, a_{n}^{p+n q}\right\},
$$

where $p \in \mathbb{N}_{0}, q \in \mathbb{Z}$ and $1 \leqslant p+q$. 
4. The constant multiset is the multiset that has the form

$$
A=\left\{a_{1}^{q}, a_{2}^{q}, \ldots, a_{n}^{q}\right\}
$$

where $q \geqslant 1$.

5. The multiset with repetitions without restrictions is the specification that has the form

$$
A=\left\{a_{1}^{\infty}, a_{2}^{\infty}, \ldots, a_{n}^{\infty}\right\} .
$$

Finally, we give an example of another class of multisets such that a number of $m$-submultisets is calculated relatively simply

$$
A=\left\{a_{1}^{2^{k_{1}}-1}, a_{2}^{2^{k_{2}}-1}, \ldots, a_{n}^{2^{k_{n}}-1}\right\}, \quad k_{1} \leqslant k_{2} \leqslant \ldots \leqslant k_{n} .
$$

\section{Number of $m$-submultisets of a multiset}

Definition 1. The set

$$
C_{m}(A)=\{B \subseteq A:|B|=m\}
$$

of all $m$-submultisets of the multiset $A=\left\{a_{1}^{k_{1}}, \ldots, a_{n}^{k_{n}}\right\}$ is called the set of $m$-combinations of elements of this multiset.

To denote the cardinality of set (20) we use the notation

$$
\left|C_{m}(A)\right|=\left(\begin{array}{c}
k_{1} k_{2} \ldots k_{n} \\
m
\end{array}\right)
$$

which was proposed in [7].

For some specifications of the multiset $A$ the cardinality of the set has been considered formerly. In particular, for $n$-element sets the classical formula

$$
(\underbrace{1 \ldots 1}_{\begin{array}{c}
n \\
m
\end{array}})=\frac{n !}{m !(n-m) !}
$$

is known.

For a multiset with repetitions without restrictions it is known that the formula

$$
(\underbrace{\infty \infty \ldots \infty}_{\begin{array}{c}
n \\
m
\end{array}})=\frac{(n+m-1) !}{m !(n-1) !}
$$

is valid.

The following theorem is proved in [16], but due to its inaccessibility to an English-speaking reader, we present its proof below.

Theorem 1. The number of $m$-submultisets of the multiset $A=\left\{a_{1}^{k_{1}}, \ldots, a_{n}^{k_{n}}\right\}$ is equal to

$$
\left(\begin{array}{c}
k_{1} k_{2} \ldots k_{n} \\
m
\end{array}\right)=\left|C_{m}(A)\right|=\sum_{\lambda \in \Lambda_{m}(A)} \prod_{j=1}^{s}\left(\begin{array}{c}
\overline{k_{j}}-\sum_{i=j+1}^{s} \lambda_{i} \\
\lambda_{j}
\end{array}\right),
$$

where $\Lambda_{m}(A)$ is the set of those solutions of the equation

$$
\sum_{i=1}^{s} i \lambda_{i}=m
$$


that satisfy the inequalities

$$
\sum_{i=j}^{s} \lambda_{i} \leqslant \overline{k_{j}}, \quad j=1, \ldots, s,
$$

where $s=\min (m, r), r=\max \left\{k_{i}\right\}, i=1, \ldots, n$, and $\overline{k_{j}}$ is the $j$ th element of specification (4), which is adjoint to the primary specification of the multiset $A$.

Proof. From the definition of the set $\Lambda_{m}(A)$ it follows that this set satisfies the conditions:

(1) $\forall B \in C_{m}(A) \Rightarrow B^{\prime \prime} \in \Lambda_{m}(A)$;

(2) $\forall \lambda \in \Lambda_{m}(A) \Rightarrow \exists B \in C_{m}(A): B^{\prime \prime}=\lambda$.

Let us prove that the set $\Lambda_{m}(A)$ consists of all integer non-negative solutions of equation (23) that satisfy inequalities (24). Indeed, let $B$ be some multiset that belongs to the set (20) and $\lambda=\left\{\lambda_{1}, \lambda_{2}, \ldots, \lambda_{p}\right\}=B^{\prime}$. Since $|B|=m$, it is obvious that the elements of this secondary specification satisfy equation (23). The truth of inequalities (24) for solutions of this equation follows from the inequalities $k_{x}(B) \leqslant k_{x}(A), x \in[B]$, where the symbol $k_{x}(B)$ denotes the multiplicity of occurrence of the element $x$ to the multiset $B$.

Let $\lambda=\left\{\lambda_{1}, \ldots, \lambda_{s}\right\}$ be some solution of equation (23) that satisfies inequalities (24). We construct a multiset $B \in C_{m}(A)$ such that $B^{\prime \prime}=\lambda$. Let us start by selecting $\lambda_{s}$ different groups of $s$ identical elements from the multiset $A$. This can always be done because $\lambda_{s} \leqslant \overline{k_{s}}$ due to (4). Suppose we have already selected $\sum_{i=j+1}^{s} \lambda_{i}$ different groups of elements such that each group consists of at least $j+1$ identical elements. Let $\overline{k_{j}}$ be the maximum number of groups of $j$ identical elements that can be selected from the multiset $A$. Then there are

$$
\overline{k_{j}}-\sum_{i=j+1}^{s} \lambda_{i}
$$

groups of $j$ identical elements in each group, in addition to other groups, in the multiset $A$ after selecting from this multiset of the above groups of elements. Thus, the selection of the following $\lambda_{j}$ groups of identical elements from the multiset $A$ ensures the fulfillment of inequalities (24).

If every secondary specification from the set $\Lambda_{m}(A)$ is assigned a non-empty set

$$
C_{m}^{\lambda}(A)=\left\{B \in C_{m}(A): B^{\prime \prime}=\left\{\lambda_{1}, \ldots, \lambda_{s}\right\}\right\}
$$

of the multisets from the set $C_{m}(A)$, then set (25) for $\lambda \in \Lambda_{m}(A)$ forms a partition of the set $C_{m}(A)$. Under this condition the equality

$$
\left|C_{m}(A)\right|=\sum_{\lambda \in \Lambda_{m}(A)}\left|C_{m}^{\lambda}(A)\right|
$$

is valid. Let us find the cardinality of set (25). It has already been determined such that the multiset $A$ contains

$$
\overline{k_{j}}-\sum_{i=j+1}^{s} \lambda_{i}
$$

groups of $j$ identical elements after selecting from the multiset $A$ of all groups of identical elements that consist of at least $j+1$ identical elements. Therefore, there are exactly

$$
\left(\begin{array}{c}
\overline{k_{j}}-\sum_{i=j+1}^{s} \lambda_{i} \\
\lambda_{j}
\end{array}\right)
$$


different choices for these groups from the multiset $A$. The number of all elements belonging to the set (25) is equal to

$$
\left|C_{m}^{\lambda}(A)\right|=\prod_{j=1}^{s}\left(\begin{array}{c}
\overline{k_{j}}-\sum_{i=j+1}^{s} \lambda_{i} \\
\lambda_{j}
\end{array}\right)
$$

by the combinatorial rule of the product. Here and then we have $\sum_{i>s}^{s} \lambda_{i}=0$. Note that if the inequalities $k_{1} \geqslant k_{2} \geqslant \ldots \geqslant k_{n}$ are fulfilled, then the elements of specification $\overline{k(A)}$, in addition to the relation (4), can be calculated accordingly to one of the following formulas:

$$
\begin{gathered}
\overline{k_{j}}=n-k^{-1}(j)+1, \quad j=1, \ldots, k_{n}, \\
\overline{k_{j}}=\sum_{i=j}^{k_{n}} \lambda_{i}, \quad j=1, \ldots, k_{n},
\end{gathered}
$$

where $\lambda_{i} \in A^{\prime \prime}$,

$$
k^{-1}(j)=\min \left\{i: k_{i} \geqslant j\right\}
$$

is the minimum preimage of those elements of the primary specification $A^{\prime}$ that are not less than $j$. Formula (29) follows from relation (14). Now from (26) and (27) it follows that formula (21) is valid.

Example 1. Calculate the number of all 6-submultisets of the multiset

$$
A=\left\{a_{1}^{5}, a_{2}^{5}, a_{3}^{5}, a_{4}^{3}, a_{5}^{3}, a_{6}^{3}, a_{7}^{3}, a_{8}^{2}, a_{9}^{2}, a_{10}^{1}, a_{11}^{1}, a_{12}^{1}, a_{13}^{1}\right\} .
$$

Here $n=13, m=6, r=5, s=\min (5,6)=5$. We get the elements of the specification $\overline{k(A)}$ from relations (4):

$$
\overline{k_{1}}=13, \overline{k_{2}}=9, \overline{k_{3}}=7, \overline{k_{4}}=3, \overline{k_{5}}=3 \text {. }
$$

To find the elements of the set $\Lambda_{m}(A)$ we seek all solutions of the equation

$$
\lambda_{1}+2 \lambda_{2}+3 \lambda_{3}+4 \lambda_{4}+5 \lambda_{5}=6 .
$$

There are ten solutions of this equation:

$$
\begin{aligned}
& (6,0,0,0,0),(4,1,0,0,0),(3,0,1,0,0),(2,2,0,0,0),(2,0,0,1,0), \\
& (1,1,1,0,0),(1,0,0,0,1),(0,3,0,0,0),(0,1,0,1,0),(0,0,2,0,0) .
\end{aligned}
$$

Moreover, all these solutions satisfy the inequalities

$$
\begin{gathered}
\lambda_{1}+\lambda_{2}+\lambda_{3}+\lambda_{4}+\lambda_{5} \leqslant 13, \quad \lambda_{2}+\lambda_{3}+\lambda_{4}+\lambda_{5} \leqslant 9, \\
\lambda_{3}+\lambda_{4}+\lambda_{5} \leqslant 7, \quad \lambda_{4}+\lambda_{5} \leqslant 3, \quad \lambda_{5} \leqslant 3 .
\end{gathered}
$$

For each solution of equation (31) we calculate the product (27) and seek the sum of these products:

$$
\begin{aligned}
C_{6}(A)= & \left(\begin{array}{c}
13 \\
6
\end{array}\right)+\left(\begin{array}{c}
12 \\
4
\end{array}\right)\left(\begin{array}{l}
9 \\
1
\end{array}\right)+\left(\begin{array}{c}
12 \\
3
\end{array}\right)\left(\begin{array}{l}
7 \\
1
\end{array}\right)+\left(\begin{array}{l}
11 \\
2
\end{array}\right)\left(\begin{array}{l}
9 \\
2
\end{array}\right)+\left(\begin{array}{c}
12 \\
2
\end{array}\right)\left(\begin{array}{l}
3 \\
1
\end{array}\right) \\
& +\left(\begin{array}{c}
11 \\
1
\end{array}\right)\left(\begin{array}{l}
8 \\
1
\end{array}\right)\left(\begin{array}{l}
7 \\
1
\end{array}\right)+\left(\begin{array}{c}
12 \\
1
\end{array}\right)\left(\begin{array}{l}
3 \\
1
\end{array}\right)+\left(\begin{array}{l}
9 \\
3
\end{array}\right)+\left(\begin{array}{l}
8 \\
1
\end{array}\right)\left(\begin{array}{l}
3 \\
1
\end{array}\right)+\left(\begin{array}{l}
7 \\
2
\end{array}\right) \\
= & 1716+4455+1540+1980+198+616+36+84+24+21=10670 .
\end{aligned}
$$

Now we calculate the number of all $m$-submultisets of the multiset whose primary specification is a positive integer function of a natural argument (16). 
Theorem 2. Suppose the multiset $A=\left\{a_{1}^{k_{1}}, \ldots, a_{n}^{k_{n}}\right\}$ has the primary specification of the form $A^{\prime}=\{g(1), g(2), \ldots, g(n)\}$ and $g(i) \geqslant i, i=1,2, \ldots, n$, then the equality

$$
\left|C_{m}(A)\right|=\sum_{\lambda_{1}+\ldots+m \lambda_{m}=m} \prod_{j=1}^{m}\left(\begin{array}{c}
n-g^{-1}(j)-\sum_{i=j+1}^{m} \lambda_{i}+1 \\
\lambda_{j}
\end{array}\right)
$$

is fulfilled for $m \leqslant n$, where $g^{-1}(j)=\min \{i: g(i) \geqslant j\}, j=1, \ldots, m$.

Proof. First note that since the inequalities $g(n) \geqslant n \geqslant m$ hold, we have $s=\min (m, g(n))=m$. Therefore equation (23) and inequalities (24) have the form

$$
\begin{gathered}
\lambda_{1}+\ldots+m \lambda_{m}=m, \\
\sum_{i=j}^{m} \lambda_{i} \leqslant \overline{k_{j}}, \quad j=1, \ldots, m .
\end{gathered}
$$

We prove that each solution of equation (33) satisfies inequalities (34). By $\Lambda$ denote the set of solutions of equation (33). From the obvious inequalities

$$
\sum_{i=j}^{m} \lambda_{i} \leqslant \max _{\Lambda}\left\{\sum_{i=j}^{m} \lambda_{i}\right\} \leqslant\left\lfloor\frac{m}{j}\right\rfloor, \quad \min \{i: g(i) \geqslant j\} \leqslant j, \quad j=1, \ldots, m,
$$

it follows that to prove the statement it is enough to prove the validity of inequalities

$$
\left\lfloor\frac{m}{j}\right\rfloor \leqslant n-j+1, \quad j=1, \ldots, m .
$$

Inequalities (35) can be proved by induction on $n$.

Thus from (28) and (30) it follows that equality (32) holds true due to Theorem 1.

Example 2. Suppose $A=\left\{a_{1}^{1}, a_{2}^{3}, a_{3}^{5}, a_{4}^{7}, a_{5}^{9}\right\}$, then $g(i)=2 i-1 \geqslant i$. We shall find $C_{4}(A)$. Here $n=5, m=4$ and the equation $\lambda_{1}+2 \lambda_{2}+3 \lambda_{3}+4 \lambda_{4}=4$ has 5 solutions:

$$
(4,0,0,0),(2,1,0,0),(1,0,1,0),(0,2,0,0),(0,0,0,1) \text {. }
$$

We have

$$
\begin{aligned}
& g^{-1}(1)=\min \{i: 2 i-1 \geqslant 1\}=1, \quad g^{-1}(2)=\min \{i: 2 i-1 \geqslant 2\}=2 \\
& g^{-1}(3)=\min \{i: 2 i-1 \geqslant 3\}=2, \quad g^{-1}(4)=\min \{i: 2 i-1 \geqslant 4\}=3
\end{aligned}
$$

Therefore,

$$
\begin{aligned}
\left|C_{4}(A)\right| & =\sum_{\lambda_{1}+2 \lambda_{2}+3 \lambda_{3}+4 \lambda_{4}=4} \prod_{j=1}^{4}\left(\begin{array}{c}
n-g^{-1}(j)-\sum_{i=j+1}^{4} \lambda_{i}+1 \\
\lambda_{j}
\end{array}\right) \\
& =\left(\begin{array}{l}
5 \\
4
\end{array}\right)+\left(\begin{array}{l}
4 \\
2
\end{array}\right)\left(\begin{array}{l}
4 \\
1
\end{array}\right)+\left(\begin{array}{l}
4 \\
1
\end{array}\right)\left(\begin{array}{l}
4 \\
1
\end{array}\right)+\left(\begin{array}{l}
4 \\
2
\end{array}\right)+\left(\begin{array}{l}
3 \\
1
\end{array}\right)=5+24+16+6+3=54 .
\end{aligned}
$$

If the primary specification of a multiset is given by some continuous function $f(x)$, then the following theorem is useful for calculation of the number of all its $m$-submultisets. 
Theorem 3. Suppose the primary specification of the multiset $A=\left\{a_{1}^{k_{1}}, \ldots, a_{n}^{k_{n}}\right\}$ has the form $A^{\prime}=\{\lfloor f(1)\rfloor,\lfloor f(2)\rfloor, \ldots,\lfloor f(n\rfloor\}$, where $f: D \rightarrow E, D=[1, n], E \supseteq[1,\lfloor f(n)\rfloor]$, is some continuous increasing function. Then the formula

$$
\left|C_{m}(A)\right|=\sum_{\lambda_{1}+\ldots+m \lambda_{m}=m} \prod_{j=1}^{m}\left(\begin{array}{c}
\left\lfloor n-\max \left(f^{-1}(j), 1\right)\right\rfloor-\sum_{i=j+1}^{m} \lambda_{i} \\
\lambda_{j}
\end{array}\right)
$$

is fulfilled for $m \leqslant n$.

Proof. Since the function $f$ is continuous and increases in its domain, we see that the equality $\min \{i: f(i) \geqslant j\}=f^{-1}(j)$ holds true for all $j \geqslant 1$. Hence, we obtain the equality

$$
\min \{i:\lfloor f(i)\rfloor \geqslant j\}= \begin{cases}\max \left(1, f^{-1}(j)\right), & f^{-1}(j) \in Z_{D}, \\ \max \left(1,\left\lfloor f^{-1}(j)\right\rfloor+1\right), & f^{-1}(j) \neq Z_{D}\end{cases}
$$

where $Z_{D}=D \cap N$. Therefore the equality $n-\min \{i:\lfloor f(i)\rfloor \geqslant j\}=\left\lfloor n-\max \left(1, f^{-1}(j)\right)\right\rfloor$ is valid and we have the equality $\overline{k_{j}}=n-\min \left\{i: k_{i} \geqslant j\right\}+1=\left\lfloor n-\max \left(1, f^{-1}(j)\right)\right\rfloor+1$.

Since the inequalities

$$
\max _{\lambda_{1}+\ldots+m \lambda_{m}=m}\left(\lambda_{j}+\ldots+\lambda_{m}\right) \leqslant\left\lfloor\frac{m}{j}\right\rfloor
$$

and $f^{-1}(j) \leqslant j$ are fulfilled for all $j=1, \ldots, m$, we see that inequality (34) is equivalent to inequality (35). The proof of this theorem is finished with similar reasoning to the reasoning over the proof of Theorem 2.

Example 3. Suppose in the multiset $A=\left\{a_{1}^{k_{1}}, a_{2}^{k_{2}}, a_{3}^{k_{3}}, a_{4}^{k_{4}}, a_{5}^{k_{5}}, a_{6}^{k_{6}}\right\}$ the primary specification is given by the continuous function $f(x)=\sqrt{x}$ on the interval $[1,6]$, i.e. $k_{i}=\lfloor\sqrt{i}\rfloor$, $i=1,2,3,4,5,6$. Then

$$
k_{1}=1, k_{2}=1, k_{3}=1, k_{4}=2, k_{5}=2, k_{6}=2 .
$$

Find, for example, the number of all 5-submultisets of the given multiset. We have 7 solutions of the equation $\lambda_{1}+2 \lambda_{2}+3 \lambda_{3}+4 \lambda_{4}+5 \lambda_{5}=5$ :

$$
(5,0,0,0,0),(3,1,0,0,0),(2,0,1,0,0),(1,2,0,0,0),(1,0,0,1,0),(0,1,1,0,0),(0,0,0,0,1) .
$$

Since $f^{-1}(x)=\min \{i: f(i) \geqslant j\}=j^{2}$, we have $\left\lfloor n-\max \left(f^{-1}(j), 1\right)\right\rfloor=n-j^{2}$. Therefore,

$$
\left|C_{5}(A)\right|=\sum_{\lambda_{1}+\ldots+5 \lambda_{5}=5} \prod_{j=1}^{m}\left(\begin{array}{c}
7-j^{2}-\sum_{i=j+1}^{5} \lambda_{i} \\
\lambda_{j}
\end{array}\right) .
$$

In the last sum each summand is corresponded to each of the seven solutions of the above equation. Moreover, only those summands are non-zero that are corresponded to the first, second and fourth solutions of the above equation. Thus,

$$
\left|C_{5}(A)\right|=\left(\begin{array}{l}
6 \\
5
\end{array}\right)+\left(\begin{array}{l}
5 \\
3
\end{array}\right)\left(\begin{array}{l}
3 \\
1
\end{array}\right)+\left(\begin{array}{l}
4 \\
1
\end{array}\right)\left(\begin{array}{l}
3 \\
3
\end{array}\right)=6+30+12=48
$$

Consider the case of a linear multiset. 
Theorem 4. Suppose $A$ is a linear multiset with the primary specification $k(A)=\{p i+q$ : $i=1, \ldots, n\}$, where $1 \leqslant p+q, q \in Z, p \in N_{0}$, then we have

$$
\left|C_{m}(A)\right|=\sum_{\lambda \in \Lambda_{m}(A)} \prod_{j=1}^{s}\left(\begin{array}{c}
\lfloor n-\max (1,(j-q) / p)\rfloor+1-\sum_{i=j+1}^{s} \lambda_{i} \\
\lambda_{j}
\end{array}\right), \quad p \neq 0,
$$

where $s=\min (m, p n+q)$.

If $m \leqslant n$, then equality (36) have the form

$$
\left|C_{m}(A)\right|=\sum_{\lambda_{1}+\ldots+s \lambda_{s}=m} \prod_{j=1}^{s}\left(\begin{array}{c}
\lfloor n-\max (1,(j-q) / p)\rfloor+1-\sum_{i=j+1}^{s} \lambda_{i} \\
\lambda_{j}
\end{array}\right), \quad p \neq 0 .
$$

Proof. Consider first the case $p \neq 0$. Since the linear function $f(i)=p i+q$ satisfies the conditions of Theorem 3, we have

$$
\overline{k_{j}}=\left\lfloor n-\max \left(1, \frac{j-q}{p}\right)\right\rfloor+1
$$

Hence equality (36) is valid.

In addition, suppose that $m \leqslant n$. Then, using equality (38) and inequality $p x+q \geqslant x$, $x \in[1, n]$, from Theorem 1 it follows equality (37).

Theorem 5. The number of m-submultisets of the constant multiset $A=\left\{a_{1}^{q}, a_{2}^{q}, \ldots, a_{n}^{q}\right\}$ can be obtained by the following formulas:

(1)

$$
\left|C_{m}(A)\right|=\sum_{\lambda \in \Lambda_{m}(A)} \frac{n !}{\lambda_{1} ! \cdot \ldots \cdot \lambda_{r} !\left(n-\lambda_{1}-\ldots-\lambda_{r}\right) !}, \quad \text { where } r=\min (m, q) ;
$$

(2) if $m \leqslant q$, then

$$
\left|C_{m}(A)\right|=\sum_{\lambda_{1}+2 \lambda_{2}+\ldots+m \lambda_{m}=m} \frac{n !}{\lambda_{1} ! \cdot \ldots \cdot \lambda_{m} !\left(n-\lambda_{1}-\ldots-\lambda_{m}\right) !} ;
$$

(3) if $m \leqslant n$, then

$$
\left|C_{m}(A)\right|=\sum_{\lambda_{1}+\ldots+s \lambda_{s}=m} \frac{n !}{\lambda_{1} ! \cdot \ldots \cdot \lambda_{s} !\left(n-\lambda_{1}-\ldots-\lambda_{s}\right) !}, \quad \text { where } s=\min (m, q) .
$$

Proof. (1) In the case of a constant multiset we have $s=\min (m, q)$ and $\overline{k_{j}}=n, j=1,2, \ldots, s$. Therefore,

$$
\left|C_{m}(A)\right|=\sum_{\lambda \in \Lambda_{m}(A)} \prod_{j=1}^{s}\left(\begin{array}{c}
n-\sum_{i=j+1}^{s} \lambda_{i} \\
\lambda_{j}
\end{array}\right)=\sum_{\lambda \in \Lambda_{m}(A)} \frac{n !}{\lambda_{1} ! \cdot \ldots \cdot \lambda_{s} !\left(n-\lambda_{1}-\ldots-\lambda_{s}\right) !} .
$$

(2) If $m \leqslant q$, then the set $\Lambda_{m}(A)$ coincides with the set of all solutions of the equation

$$
\lambda_{1}+2 \lambda_{2}+\ldots+m \lambda_{m}=m \text {. }
$$

Therefore formula (39) has the form (40).

(3) If $m \leqslant n$, then inequalities (24) hold for all $j=1, \ldots, s$ and equality (41) is valid.

Remark 2. From Theorem 5 (see item (2)) and equality (22) it follows that

$$
\sum_{\lambda_{1}+2 \lambda_{2}+\ldots+m \lambda_{m}=m} \frac{n !}{\lambda_{1} ! \cdot \ldots \cdot \lambda_{m} !\left(n-\lambda_{1}-\ldots-\lambda_{m}\right) !}=\left(\begin{array}{c}
n+m-1 \\
m
\end{array}\right) .
$$

Notice that the left-hand side of this identity consists only of those summands such that $n-\left(\lambda_{1}+\ldots+\lambda_{m}\right) \geqslant 0$. 


\section{Generatix method}

A generatix is the function

$$
f(t)=\prod_{i=1}^{n} \sum_{j=0}^{k_{i}} t^{j}=\sum_{i=0}^{k_{1}+\ldots+k_{n}}\left|C_{i}(A)\right| t^{i}
$$

for the calculation of the number of $m$-submultisets of the multiset $A=\left\{a_{1}^{k_{1}}, \ldots, a_{n}^{k_{n}}\right\}$. Therefore, after $m$-fold differentiation of this function we obtain the equality

$$
\left|C_{m}(A)\right|=\frac{1}{m !} \frac{d^{m} f}{d t^{m}}(0) .
$$

We have

$$
\frac{d^{m} f(t)}{d t^{m}}=\sum_{r_{1}+\ldots+r_{n}=m} \frac{m !}{r_{1} ! \ldots r_{n} !} \frac{d^{r_{1}} g_{1}(t)}{d t^{r_{1}}} \ldots \frac{d^{r_{n}} g_{n}(t)}{d t^{r_{n}}},
$$

where $g_{i}(t)=1+t+t^{2}+\ldots+t^{k_{i}}$. Since

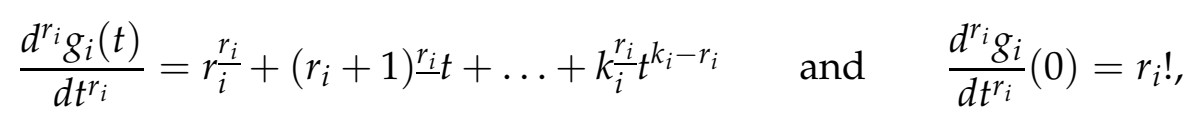

we obtain

$$
\left|C_{m}(A)\right|=\frac{1}{m !} \frac{d^{m} f}{d t^{m}}(0)=\frac{1}{m !} \sum_{r_{1}+\ldots+r_{n}=m} \frac{m !}{r_{1} ! \ldots r_{n} !} r_{1} ! \ldots r_{n} !=\sum_{r_{1}+\ldots+r_{n}=m} 1,
$$

where $0 \leqslant r_{i} \leqslant k_{i}$.

Thus, we have the next theorem.

Theorem 6. The number of m-submultisets of the multiset $A=\left\{a_{1}^{k_{1}}, \ldots, a_{n}^{k_{n}}\right\}$ is equal to

$$
\left|C_{m}(A)\right|=\sum_{\substack{r_{1}+r_{2}+\ldots+r_{n}=m \\ 0 \leqslant r_{i} \leqslant k_{i}, i=1, \ldots, n}} 1 .
$$

Let us use Theorem 6 to determine the formula for the calculation of the number of $m$-submultisets of the constant multiset $A=\left\{a_{1}^{q}, a_{2}^{q}, \ldots, a_{n}^{q}\right\}$. First note that if the solution $\left(s_{1}, s_{2}, \ldots, s_{n}\right)$ of the equation

$$
r_{1}+r_{2}+\ldots+r_{n}=m
$$

satisfies the inequalities $0 \leqslant s_{i} \leqslant q$, then an arbitrary permutation of the components of this solution leads to a new solution of this equation. Therefore we need to find all disordered solutions of equation (42), i.e. such solutions $\left(r_{1}, r_{2}, \ldots, r_{n}\right)$ that satisfy the inequalities $r_{1} \geqslant r_{2} \geqslant \ldots \geqslant r_{n} \geqslant 0$ and we need to count the number of permutations of the components of each solution. Suppose among the components of solution $\left(r_{1}, r_{2}, \ldots, r_{n}\right)$ are $\lambda_{0}$ zeros, $\lambda_{1}$ ones, and so on; then all disordered solutions of equation (42) can be counted using the system of equations

Therefore,

$$
\left\{\begin{array}{l}
0 \lambda_{0}+1 \lambda_{1}+\ldots+q \lambda_{q}=m \\
\lambda_{0}+\lambda_{1}+\ldots+\lambda_{q}=n
\end{array}\right.
$$

Thus the next theorem is valid.

$$
C_{m}(A)=\sum_{\substack{0 \lambda_{0}+1 \lambda_{1}+\ldots+q \lambda_{q}=m \\ \lambda_{0}+\lambda_{1}+\ldots+\lambda_{q}=n}} \frac{n !}{\lambda_{0} ! \lambda_{1} ! \ldots \lambda_{q} !}
$$


Theorem 7. The number of $m$-submultisets of the constant multiset $A=\left\{a_{1}^{q}, a_{2}^{q}, \ldots, a_{n}^{q}\right\}$ is equal to

$$
C_{m}(A)=\sum_{\substack{0 \lambda_{0}+1 \lambda_{1}+\ldots+q \lambda_{q}=m \\ \lambda_{0}+\lambda_{1}+\ldots+\lambda_{q}=n}} \frac{n !}{\lambda_{0} ! \lambda_{1} ! \ldots \lambda_{q} !} .
$$

Remark 3. If in Theorem $7 m \leqslant q$, then, using equality (22), we obtain the following identity

$$
\sum_{\substack{0 \lambda_{0}+1 \lambda_{1}+\ldots+m \lambda_{m}=m \\
\lambda_{0}+\lambda_{1}+\ldots+\lambda_{m}=n}} \frac{n !}{\lambda_{0} ! \lambda_{1} ! \ldots \lambda_{m} !}=\left(\begin{array}{c}
m+n-1 \\
m
\end{array}\right) .
$$

Example 4. Let us find the number of $m$-submultisets of the multiset with specification $A=\left\{x_{1}^{\infty}, x_{2}^{\infty}, \ldots, x_{n}^{\infty}\right\}$ which contain each element of basis $[A]$ (see [12]) of the multiset $A$. To find them we use the generatrix

$$
W(t)=\left(\sum_{i=1}^{\infty} t^{i}\right)^{n}=t^{n}(1-t)^{-n}=t^{n} \sum_{i=0}^{\infty} \frac{n^{\bar{i}}}{i !} t^{i}=\sum_{i=0}^{\infty} \frac{n^{\bar{i}}}{i !} t^{n+i}
$$

Put $n+i=m$, then

$$
W(t)=\sum_{m=n}^{\infty} \frac{n^{\overline{m-n}}}{(m-n) !} t^{m}=\sum_{m=n}^{\infty}\left(\begin{array}{c}
m-1 \\
n-1
\end{array}\right) t^{m} .
$$

We shall consider one more class of multisets with primary specification (19), i.e.

$$
A=\left\{a_{1}^{2^{l_{1}}-1}, \ldots, a_{n}^{2^{l_{n}}-1}\right\}, \quad l_{1} \leqslant l_{2} \leqslant \ldots \leqslant l_{n},
$$

such that their number of $m$-submultisets is calculated relatively easily. As shown in [12], the generatrix of the number of $m$-submultisets of such multisets has the form

$$
W(t)=\prod_{i=1}^{n} \sum_{j=0}^{2^{l_{i}-1}} t^{j}
$$

However $1+t+\ldots+t^{2^{l}-1}=(1+t)\left(1+t^{2}\right)\left(1+t^{4}\right) \ldots\left(1+t^{2^{l-1}}\right)$ whence, using the designation $t^{2^{i}}=x_{i+1}, i=0, \ldots, l-1$, we get

$$
W(t)=\left(1+x_{1}\right)^{m_{1}} \ldots\left(1+x_{l_{n}}\right)^{m_{n}} .
$$

Obviously, the number $\left|C_{m}(A)\right|$ is equal to the sum of coefficients of the monomials

$$
K\left(\lambda_{1}, \ldots, \lambda_{l_{n}}\right) x_{1}^{\lambda_{1}} \ldots x_{l_{n}}^{\lambda_{l_{n}}}
$$

with $l_{n}$ variables such that their indices $\lambda_{1}, \lambda_{2}, \ldots, \lambda_{l_{n}}$ are the components of solutions of the equation

$$
\lambda_{1}+2 \lambda_{2}+2^{2} \lambda_{3}+\ldots+2^{l_{n}-1} \lambda_{l_{n}}=m
$$

and these indices satisfy the inequalities $\lambda_{i} \leqslant \overline{k_{2^{i-1}}}, i=1, \ldots, l_{n}$, where $\left(\overline{k_{1}}, \ldots, \overline{k_{2} l_{n-1}}\right)$ is the specification of the multiset $\bar{A}$ that is adjoint to the multiset $A$. Therefore,

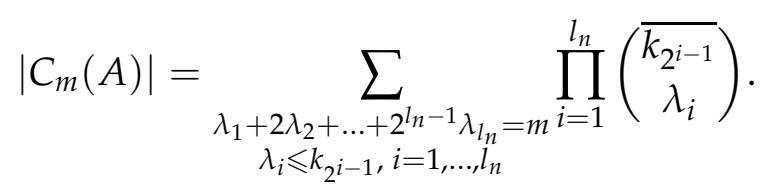

Thus the next theorem is valid. 
Theorem 8. The number of m-submultisets of the multiset

$$
A=\left\{a_{1}^{2_{1}^{l_{1}}-1}, \ldots, a_{n}^{2^{l_{n}}-1}\right\}, \quad l_{1} \leqslant l_{2} \leqslant \ldots \leqslant l_{n}
$$

is equal to

$$
\left|C^{m}(A)\right|=\sum_{\substack{\lambda_{1}+2 \lambda_{2}+\ldots+2^{l_{n}-1} \lambda_{l_{n}}=m \\
\lambda_{i} \leqslant \overline{k_{2} i-1}, i=1, \ldots, l_{n}}} \prod_{i=1}^{l_{n}}\left(\begin{array}{c}
\overline{k_{2^{i-1}}} \\
\lambda_{i}
\end{array}\right) .
$$

Example 5. Suppose we have the multiset $A=\left\{a_{1}^{3}, a_{2}^{7}, a_{3}^{15}, a_{4}^{31}\right\}$. We seek the primary specification of the adjoint multiset $\bar{A}$ :

$$
\bar{k}=(4,4,4,3,3,3,3,2,2,2,2,2,2,2,2,1,1,1,1,1,1,1,1,1,1,1,1,1,1,1,1) .
$$

Therefore,

The equation

$$
\overline{k_{1}}=4, \overline{k_{2}}=4, \overline{k_{4}}=3, \overline{k_{8}}=2, \overline{k_{16}}=1
$$

$$
\lambda_{1}+2 \lambda_{2}+4 \lambda_{3}+8 \lambda_{4}+16 \lambda_{5}=21
$$

have 60 solutions. But only 12 solutions satisfy the inequalities

$$
\lambda_{1} \leqslant \overline{k_{1}}, \lambda_{2} \leqslant \overline{k_{2}}, \lambda_{3} \leqslant \overline{k_{4}}, \lambda_{4} \leqslant \overline{k_{8}}, \lambda_{5} \leqslant \overline{k_{16}} .
$$

List of these solutions:

$$
\begin{aligned}
& (3,3,3,0,0),(3,3,1,1,0),(3,1,2,1,0),(3,1,0,2,0),(3,1,0,0,1),(1,4,1,1,0), \\
& (1,2,2,1,0),(1,2,0,2,1),(1,2,0,0,1),(1,0,3,1,0),(1,0,1,2,0),(1,0,1,0,1) .
\end{aligned}
$$

Thus, we have

$$
\begin{aligned}
&\left|C^{21}(A)\right|=\left(\begin{array}{l}
4 \\
3
\end{array}\right)\left(\begin{array}{l}
4 \\
3
\end{array}\right)\left(\begin{array}{l}
3 \\
3
\end{array}\right)+\left(\begin{array}{l}
4 \\
3
\end{array}\right)\left(\begin{array}{l}
4 \\
3
\end{array}\right)\left(\begin{array}{l}
3 \\
1
\end{array}\right)\left(\begin{array}{l}
2 \\
1
\end{array}\right)+\left(\begin{array}{l}
4 \\
3
\end{array}\right)\left(\begin{array}{l}
4 \\
1
\end{array}\right)\left(\begin{array}{l}
3 \\
2
\end{array}\right)\left(\begin{array}{l}
2 \\
1
\end{array}\right)+\left(\begin{array}{l}
4 \\
3
\end{array}\right)\left(\begin{array}{l}
4 \\
1
\end{array}\right)\left(\begin{array}{l}
2 \\
2
\end{array}\right) \\
&+\left(\begin{array}{l}
4 \\
3
\end{array}\right)\left(\begin{array}{l}
4 \\
1
\end{array}\right)\left(\begin{array}{l}
1 \\
1
\end{array}\right)+\left(\begin{array}{l}
4 \\
1
\end{array}\right)\left(\begin{array}{l}
4 \\
4
\end{array}\right)\left(\begin{array}{l}
3 \\
1
\end{array}\right)\left(\begin{array}{l}
2 \\
1
\end{array}\right)+\left(\begin{array}{l}
4 \\
1
\end{array}\right)\left(\begin{array}{l}
4 \\
2
\end{array}\right)\left(\begin{array}{l}
3 \\
2
\end{array}\right)\left(\begin{array}{l}
2 \\
1
\end{array}\right)+\left(\begin{array}{l}
4 \\
1
\end{array}\right)\left(\begin{array}{l}
4 \\
2
\end{array}\right)\left(\begin{array}{l}
2 \\
2
\end{array}\right)\left(\begin{array}{l}
1 \\
1
\end{array}\right) \\
&+\left(\begin{array}{l}
4 \\
1
\end{array}\right)\left(\begin{array}{l}
4 \\
2
\end{array}\right)\left(\begin{array}{l}
1 \\
1
\end{array}\right)+\left(\begin{array}{l}
4 \\
1
\end{array}\right)\left(\begin{array}{l}
3 \\
3
\end{array}\right)\left(\begin{array}{l}
2 \\
1
\end{array}\right)+\left(\begin{array}{l}
4 \\
1
\end{array}\right)\left(\begin{array}{l}
3 \\
1
\end{array}\right)\left(\begin{array}{l}
2 \\
2
\end{array}\right)+\left(\begin{array}{l}
4 \\
1
\end{array}\right)\left(\begin{array}{l}
3 \\
1
\end{array}\right)\left(\begin{array}{l}
1 \\
1
\end{array}\right)=488 .
\end{aligned}
$$

\section{Algorithm for calculation of $m$-submultisets of an arbitrary multiset}

Let us construct a recursive algorithm for calculation of the number of $m$-submultisets of the multiset $A=\left\{a_{1}^{k_{1}}, a_{2}^{k_{2}}, \ldots, a_{n}^{k_{n}}\right\}$.

We use for the number $C_{m}(A)$ the notation from [7]. Then we have

$$
\prod_{i=1}^{n}\left(1+t+t^{2}+\ldots+t^{k_{i}}\right)=\sum_{i=0}^{r}\left(\begin{array}{c}
k_{1}, k_{2}, \ldots, k_{n} \\
i
\end{array}\right) t^{i}, \quad r=\sum_{i=1}^{n} k_{i} .
$$

If the coefficients

of the polynomial

$$
A(i)=\left(\begin{array}{c}
k_{1}, k_{2}, \ldots, k_{l-1} \\
i
\end{array}\right), \quad i=0, \ldots, s,
$$

$$
\sum_{i=0}^{s} A(i) t^{i}=\prod_{i=1}^{l-1}\left(1+t+\ldots+t^{k_{i}}\right), \quad s=\sum_{i=1}^{l-1} k_{i},
$$


are known, then the coefficients

$$
B(j)=\left(\begin{array}{c}
k_{1}, k_{2}, \ldots, k_{l} \\
j
\end{array}\right), \quad j=0,1, \ldots, s+k_{l},
$$

of the polynomial

$$
\sum_{j=0}^{s+k_{l}} B(j) t^{j}=\left(1+t+\ldots+t^{k_{l}}\right) \sum_{i=0}^{s} A(i) t^{i}
$$

are obtained by summing $k_{l}+1$ last elements of the row

$$
\underbrace{0 \ldots 0}_{k_{l}} A(0) A(1) \ldots A(j) \underbrace{0 \ldots 0}_{k_{l}} \text {. }
$$

More exactly, we have

$$
\left(\begin{array}{c}
k_{1}, k_{2}, \ldots, k_{l} \\
j
\end{array}\right)=\sum_{q=j-k_{l}}^{j}\left(\begin{array}{c}
k_{1}, k_{2}, \ldots, k_{l-1} \\
q
\end{array}\right), \quad j=0, \ldots, s+k_{l},
$$

where

if $q<0$ or $q>s$.

$$
\left(\begin{array}{c}
k_{1}, k_{2}, \ldots, k_{l-1} \\
q
\end{array}\right)=0
$$

The calculation process is convenient to design according to the next algorithm.

Algorithm 1 ([17]). We calculate $C_{m}(A)$ if $A=\left\{a_{1}^{k_{1}}, a_{2}^{k_{2}}, \ldots, a_{n}^{k_{n}}\right\}$.

We construct the algorithm in the form of a table of elements $A(i, j)$, where $i$ is the row number, and $j$ is the column number of the table. The elements of non-negative columns are called the significant elements.

Step $1 . s:=0, i:=1, j:=0$.

Step 2. We specify the significant element of the first line: $A(i, j):=1$.

Step 3. While $i<n+1$ go to step 4, otherwise issue result is $C_{m}(A)=A(i, m)$ and stop work.

Step 4. Add to the left and right of the significant elements of $i$ th row in $k_{i}$ zeros: for $j$ from $s-k_{i}$ to $s-1$ specify $A(i, j):=0$, for $j$ from $s+1$ to $s+k_{i}$ specify $A(i, j):=0$.

Step 5. Find the significant elements of the $(i+1)$ th row: for $p$ from 0 to $s+k_{i}$ execute $A(i+1, p):=\sum_{j=p-k_{i}}^{p} A(i, j)$.

Step 6. $s:=s+k_{i}, i:=i+1$, and go to Step 3 .

We illustrate this algorithm in the form of the table.

Example 6. Suppose we have the multiset $A=\left\{a_{1}^{3}, a_{2}^{2}, a_{3}^{2}\right\}$, then, using the above algorithm, we obtain the following table.

$$
\begin{array}{ccccccccccc} 
& & & C_{0}(A) & C_{1}(A) & C_{2}(A) & C_{3}(A) & C_{4}(A) & C_{5}(A) & C_{6}(A) & C_{7}(A) \\
0 & 0 & 0 & 1 & 0 & 0 & 0 & & & & \\
0 & 0 & 1 & 1 & 1 & 1 & 0 & 0 & & \\
0 & 0 & 1 & 2 & 3 & 3 & 2 & 1 & 0 & 0 \\
& & \mathbf{1} & \mathbf{3} & \mathbf{6} & \mathbf{8} & \mathbf{8} & \mathbf{6} & \mathbf{3} & \mathbf{1}
\end{array}
$$

The results are written in the last row of this table: $C_{0}(A)=1, C_{1}(A)=3, C_{2}(A)=6$, $C_{3}(A)=8, \ldots$ 
Remark 4. If the multiplicities of the multiset elements are large, then it is convenient to use the relations

$$
\left(\begin{array}{c}
k_{1}, k_{2}, \ldots, k_{l} \\
j
\end{array}\right)=\left(\begin{array}{c}
k_{1}, k_{2}, \ldots, k_{l} \\
j-1
\end{array}\right)+\left(\begin{array}{c}
k_{1}, k_{2}, \ldots, k_{l-1} \\
j
\end{array}\right)-\left(\begin{array}{c}
k_{1}, k_{2}, \ldots, k_{l-1} \\
j-k_{l}-1
\end{array}\right), \quad j=0, \ldots, \sum_{i=1}^{l} k_{i},
$$

which follow from relations (43). This can significantly reduce the number of operations.

Remark 5. Since any $k$-submultiset $B$ of the multiset $A$ uniquely corresponds to $(|A|-k)$ submultiset $A-B$ of this multiset, we have

$$
\left(\begin{array}{c}
k_{1}, k_{2}, \ldots, k_{l} \\
j
\end{array}\right)=\left(\begin{array}{c}
k_{1}, k_{2}, \ldots, k_{l} \\
s-j
\end{array}\right), \quad j=0, \ldots, s,
$$

where $s=\sum_{i=1}^{l} k_{i}$, i.e. the numbers that are equidistant from the ends of each row of the table are equal to each other. Thus, if $m>\lfloor s / 2\rfloor$, then instead of calculating $\left|C_{m}(A)\right|$ it is more convenient to calculate $\left|C_{s-m}(A)\right|$.

\section{6 m-permutations of the multiset elements}

Definition 2. The set of all ordered m-samples of elements of the multiset $A=\left\{a_{1}^{k_{1}}, \ldots, a_{n}^{k_{n}}\right\}$ is called the set of m-permutations on this multiset. $B y P_{m}(A)$ we denote this set.

The following statement is well known.

Proposition 2. The number of all permutations of elements of the multiset $A$ is equal to

$$
\left|P_{|A|}(A)\right|=\frac{\left(k_{1}+k_{2}+\ldots+k_{n}\right) !}{k_{1} ! k_{2} ! \ldots k_{n} !} .
$$

To determine the number of all $m$-permutations of the multiset $A=\left\{a_{1}^{k_{1}}, \ldots, a_{n}^{k_{n}}\right\}$ we use Theorem 1 . In this theorem it was found that the number of all $m$-combinations of the multiset $A$ is equal to

where

$$
\left|C_{m}(A)\right|=\sum_{\lambda \in \Lambda_{m}(A)}\left|C_{m}^{\lambda}(A)\right|
$$

Obviously,

$$
C_{m}^{\lambda}(A)=\left\{B \in C_{m}(A): B^{\prime \prime}=\left\{\lambda_{1}, \ldots, \lambda_{n}\right\}\right\} .
$$

but

$$
\left|P_{m}(A)\right|=\sum_{\lambda \in \Lambda_{m}(A)}\left|P_{|B|}(B)\right|\left|C_{m}^{\lambda}(A)\right|
$$

whence equality (44) leads to the following theorem.

$$
\left|P_{|B|}(B)\right|=\frac{m !}{1 !^{\lambda_{1}} 2 !^{\lambda_{2}} \ldots s !^{\lambda_{s}}}
$$

Theorem 9 ([16]). The number of all m-permutations of the multiset $A=\left\{a_{1}^{k_{1}}, \ldots, a_{n}^{k_{n}}\right\}$ is equal to

$$
\left|P_{m}(A)\right|=\sum_{\lambda \in \Lambda_{m}(A)} \frac{m !}{1 !^{\lambda_{1} 2 !^{\lambda_{2}} \ldots s ! \lambda_{s}}} \prod_{j=1}^{s}\left(\begin{array}{c}
\overline{k_{j}}-\sum_{i=j+1}^{s} \lambda_{i} \\
\lambda_{j}
\end{array}\right)
$$

where $\Lambda_{m}(A)$ is the set of those solutions of the equation

$$
\sum_{i=1}^{s} i \lambda_{i}=m
$$


that satisfy the inequalities

$$
\sum_{i=j}^{s} \lambda_{i} \leqslant \overline{k_{j}}, \quad j=1, \ldots, s, \quad s=\min (m, r), \quad r=\max \left\{k_{i}\right\}, \quad i=1, \ldots, n,
$$

$\overline{k_{j}}$ is the jth element of specification (4), which is adjoint to the primary specification of the multiset $A$.

The number of solutions of the equation $\sum_{i=1}^{s} i \lambda_{i}=m$ increases with increasing $m$ and $s$. For example, already at $m=s=20$ this equation has 627 solutions. Consequently formula (45) is not always convenient for practical use because it requires large amounts of computation.

We construct an algorithm for calculating $m$-permutations of elements of the multiset $A=\left\{a_{1}^{k_{1}}, \ldots, a_{n}^{k_{n}}\right\}$ such that in many cases this algorithm eliminates these shortcomings.

Let

$$
\left|P_{m}(A)\right|=\left[\begin{array}{c}
k_{1} k_{2} \ldots k_{n} \\
m
\end{array}\right] .
$$

In particular, if $k_{1}=k_{2}=\ldots=k_{n}=1$, then the multiset coincides with its basis and this multiset is an ordinary set, i.e.

$$
\left[\begin{array}{c}
\underbrace{1 \ldots 1}_{n} \\
m
\end{array}\right]=\frac{n !}{(n-m) !}, \quad 0 \leqslant m \leqslant n .
$$

If $k_{1}=n, k_{2}=k_{3}=\ldots=k_{n}=0$, then

$$
\left[\begin{array}{l}
n \\
m
\end{array}\right]=1, \quad 0 \leqslant m \leqslant n .
$$

In the case, where the multiset $A$ has specification (18), we have the obvious equality

$$
[\underbrace{\infty \infty \cdots \infty}_{n}]=n^{m}
$$

Theorem 10. For any $r=2,3, \ldots, n$ the equality

$$
\left[\begin{array}{c}
k_{1} k_{2} \ldots k_{r} \\
i
\end{array}\right]=\left\{\begin{array}{l}
\sum_{j=0}^{\min \left(i, k_{1}+\ldots+k_{r-1}\right)}\left(\begin{array}{l}
i \\
j
\end{array}\right)\left[\begin{array}{c}
k_{1} k_{2} \ldots k_{r-1} \\
i
\end{array}\right], \quad i \leqslant k_{r} \\
\sum_{j=i-k_{r}}^{\min \left(i, k_{1}+\ldots+k_{r-1}\right)}\left(\begin{array}{l}
i \\
j
\end{array}\right)\left[\begin{array}{c}
k_{1} k_{2} \ldots k_{r-1} \\
i
\end{array}\right], \quad k_{r}<i \leqslant k_{1}+\ldots+k_{r}
\end{array}\right.
$$

is fulfilled, where $0 \leqslant i \leqslant|A|$.

Proof. The generatrix for the number of permutations

$$
\left[\begin{array}{c}
k_{1} k_{2} \ldots k_{r} \\
i
\end{array}\right]
$$

of elements of the multiset $A=\left\{a_{1}^{k_{1}}, \ldots, a_{n}^{k_{n}}\right\}$ has the form

$$
\prod_{i=1}^{n} \sum_{j=0}^{k_{i}} \frac{t^{j}}{j !}=\sum_{i=0}^{k_{1}+\ldots+k_{n}}\left[\begin{array}{c}
k_{1} k_{2} \ldots k_{n} \\
i
\end{array}\right] \frac{t^{i}}{i !}
$$

Hence,

$$
\left(\sum_{j=0}^{k_{1}+\ldots+k_{r-1}}\left[\begin{array}{c}
k_{1} k_{2} \ldots k_{r-1} \\
j
\end{array}\right] \frac{t^{j}}{j !}\right) \sum_{s=0}^{k_{r}} \frac{t^{s}}{s !}=\sum_{i=0}^{k_{1}+\ldots+k_{r}}\left[\begin{array}{c}
k_{1} k_{2} \ldots k_{r} \\
i
\end{array}\right] \frac{t^{i}}{i !}
$$


Since

$$
\left(\sum_{j=0}^{k_{1}+\ldots+k_{r-1}}\left[\begin{array}{c}
k_{1} k_{2} \ldots k_{r-1} \\
j
\end{array}\right] \frac{t^{j}}{j !}\right) \sum_{s=0}^{k_{r}} \frac{t^{s}}{s !}=\sum_{i=0}^{k_{1}+\ldots+k_{r}}\left(\sum_{j+s=i}\left[\begin{array}{c}
k_{1} k_{2} \ldots k_{r-1} \\
j
\end{array}\right] \frac{t^{i}}{j ! s !}\right)
$$

we have

$$
\left[\begin{array}{c}
k_{1} k_{2} \ldots k_{r} \\
i
\end{array}\right]=\sum_{j+s=i} \frac{i !}{j ! s !}\left[\begin{array}{c}
k_{1} k_{2} \ldots k_{r-1} \\
j
\end{array}\right]=\sum_{j+s=i}\left(\begin{array}{c}
i \\
j
\end{array}\right)\left[\begin{array}{c}
k_{1} k_{2} \ldots k_{r-1} \\
j
\end{array}\right] .
$$

In the last sum, for both expressions

$$
\left(\begin{array}{l}
i \\
j
\end{array}\right) \text { and }\left[\begin{array}{c}
k_{1} \ldots k_{r-1} \\
j
\end{array}\right]
$$

to have meaning it is necessary to have the inequalities $j \leqslant i$ and $j \leqslant k_{1}+\ldots+k_{r-1}$, i.e. the inequality $j \leqslant \min \left(i, k_{1}+\ldots+k_{r-1}\right)$ is valid. If $i \leqslant k_{r}$, then from the inequality $0 \leqslant s \leqslant k_{r}$ it follows that the smallest value of the index $j$ under the restriction $j+s=i$ is $j=0$. If $i>k_{r}$, then the smallest value of the index $j$ is $j=i-k_{r}$. This completes the proof.

Recurrence equality (47) can be used to calculate the number of all $m$-permutations of the multiset $A=\left\{a_{1}^{k_{1}}, \ldots, a_{n}^{k_{n}}\right\}$, where $m=0, \ldots,|A|$.

For this purpose, we present the following algorithm.

Algorithm 2 ([18]). Step 1. Write the row of $k_{1}+1$ ones, which are numbers of $i$-permutations

$$
\left[\begin{array}{c}
k_{1} \\
i
\end{array}\right]
$$

on the multiset $A=\left\{a_{1}^{k_{1}}\right\}, i=0, \ldots, k_{1}$. This row is called the basic row.

Step 2. Under the basic row we construct a table with $k_{1}+1$ columns and $k_{1}+k_{2}+1$ rows. We number rows of the table from top to bottom by numbers from 0 to $k_{1}+k_{2}$.

Step 3. In the ith row of the table we write the first $k_{1}+1$ elements of the ith row of the Pascal triangle. If the ith row of the Pascal triangle contains the less than $k_{1}+1$ elements, then we add the required number of zeros.

Step 4. In the lower left corner of the table we replace the written numbers by zeros so that the zeros form a right isosceles triangle with the leg $k_{1}$.

Step 5. We calculate the sum of the products of elements for the $i$ th $\left(i=0, \ldots, k_{1}+k_{2}\right)$ row of the table and the corresponding elements of the basic row. The resulting number of permutations

is added to the ith row on the right.

$$
\left[\begin{array}{c}
k_{1}, k_{2} \\
i
\end{array}\right], \quad i=0, \ldots, k_{1}+k_{2}
$$

Step 6. If the number of rows of the last table is greater than the cardinality of the multiset, then the calculation is completed and the result of the algorithm is the column of numbers such that these numbers were added to the table on the right. Otherwise, we transpose the column of numbers that were added to the table on the right, consider this as the base row of the new table, the parameters of the table are increased by the value of the multiplicity of the next element of the multiset, and then we go to Step 2.

Thus, if the multiset $A$ has the cardinality basis $n$, then the execution of the algorithm requires the construction of the $(n-1)$ th table. 
Example 7. Find the number of all m-permutations of the multiset $A=\left\{a_{1}^{2}, a_{2}^{4}, a_{3}^{5}\right\}$, $m=0,1, \ldots, 11$. For this purpose we build the following tables.

\begin{tabular}{|c|c|c|c|c|}
\hline & \multicolumn{3}{|c|}{11} \\
\hline 0 & 1 & 0 & 0 & 1 \\
\hline 1 & 1 & 1 & 0 & 2 \\
\hline 2 & 1 & 2 & 1 & 4 \\
\hline 3 & 1 & 3 & 3 & 7 \\
\hline 4 & 1 & 4 & 6 & 11 \\
\hline 5 & 0 & 5 & 10 & 15 \\
\hline 6 & 0 & 0 & 15 & 15 \\
\hline
\end{tabular}

\begin{tabular}{|c|c|c|c|c|c|c|c|c|}
\hline & 1 & 2 & 4 & 7 & 11 & 15 & 15 & \\
\hline 0 & 1 & 0 & 0 & 0 & 0 & 0 & 0 & 1 \\
\hline 1 & 1 & 1 & 0 & 0 & 0 & 0 & 0 & 3 \\
\hline 2 & 1 & 2 & 1 & 0 & 0 & 0 & 0 & 9 \\
\hline 3 & 1 & 3 & 3 & 1 & 0 & 0 & 0 & 26 \\
\hline 4 & 1 & 4 & 6 & 4 & 1 & 0 & 0 & 72 \\
\hline 5 & 1 & 5 & 10 & 10 & 5 & 1 & 0 & 191 \\
\hline 6 & 0 & 6 & 15 & 20 & 15 & 6 & 1 & 482 \\
\hline 7 & 0 & 0 & 21 & 35 & 35 & 21 & 7 & 1134 \\
\hline 8 & 0 & 0 & 0 & 56 & 70 & 56 & 28 & 2422 \\
\hline 9 & 0 & 0 & 0 & 0 & 126 & 126 & 34 & 4536 \\
\hline 10 & 0 & 0 & 0 & 0 & 0 & 252 & 210 & 6930 \\
\hline 11 & 0 & 0 & 0 & 0 & 0 & 0 & 462 & 6930 \\
\hline
\end{tabular}

Therefore,

$$
\begin{gathered}
P^{0}(A)=1, P^{1}(A)=3, P^{2}(A)=9, P^{3}(A)=26, P^{4}(A)=72, P^{5}(A)=191, P^{6}(A)=482, \\
P^{7}(A)=1134, P^{8}(A)=2422, P^{9}(A)=4536, P^{10}(A)=6930, P^{11}(A)=6930 .
\end{gathered}
$$

This algorithm is effective for multisets of relatively large cardinality but with a small base. For example, to calculate the number of 20-permutations on the multiset $A=\left\{a_{1}^{3}, a_{2}^{9}, a_{3}^{13}\right\}$ this algorithm requires the construction of two tables of sizes $4 \times 13$ and $13 \times 26$ accordingly and the calculation by the formula requires the analysis of the set of 627 solutions of equation (46) and significant calculations.

Example 8. For the multiset $A=\left\{a_{1}^{1}, a_{2}^{1}, a_{3}^{1}, a_{4}^{2}, a_{5}^{2}, a_{6}^{2}, a_{7}^{2}, a_{8}^{3}, a_{9}^{3}, a_{10}^{5}\right\}$ we have

$$
\begin{gathered}
P^{0}(A)=1, P^{1}(A)=10, P^{2}(A)=97, P^{3}(A)=912, P^{4}(A)=8299, \\
P^{5}(A)=72946, P^{6}(A)=617874, P^{7}(A)=5029948, P^{8}(A)=39237380, \\
P^{9}(A)=292327224, P^{10}(A)=2072330400, P^{11}(A)=13920355680, \\
P^{12}(A)=88179787080, P^{13}(A)=523856052720, P^{14}(A)=2899520704080, \\
P^{15}(A)=14831963546400, P^{16}(A)=6938695764000, \\
P^{17}(A)=292608485769600, P^{18}(A)=1088829613872000, \\
P^{19}(A)=3456466684070400, P^{20}(A)=8834757003072000, \\
P^{21}(A)=162615846032640000, P^{22}(A)=162615846032640000 .
\end{gathered}
$$

\section{References}

[1] Aigner M. Combinatorial theory. Springer-Verlag, New York, 1979.

[2] Barton D.E., Mallows C.L. Some aspects of the random sequence. Ann. Math. Statist. 1965, 36 (1), $236-260$. doi:10.1214/aoms/1177700286

[3] Cartier P., Foata D. Problèmes combinatoires de commutation et réarrangements. In: Dold A., Eckmann B. (Eds.) Lecture Notes in Mathematics, 85. Springer-Verlag, Berlin, 1969.

[4] David F.N., Barton D.E. Combinatorial chance. Charles Griffin \& Co. Limited, London, 1962. 
[5] Foata D. Etude algébrique de certains problèmes d'analyse combinatoire et du calcul des probabilités. Publ. Inst. Statist. Univ. Paris 1965, 14, 81-241.

[6] Gotsulenko V.V. A formula for the number of combinations with constrained repetitions and its application. Prikl. Diskretn. Mat. 2013, 20 (2), 71-77. doi:10.17223/20710410/20/8 (in Russian)

[7] Green C., Kleitman D.J. Proof techniques in the theory of finite sets. In: Rota G.-C. (Ed.) Studies in combinatorics, 17. Math. Assoc. of Amer., Washington, 1978.

[8] Greene D.H., Knuth D.E. Mathematics for the analysis of algorithms. Birkhäuser, Boston, 1990.

[9] Hage J. Enumerating submultisets of multisets. Inf. Proc. Letters 2003, 85 (4), 221-226. doi:10.1016/S00200190(02)00394-0

[10] Knuth D.E. The art of computer pragramming. Sorting and searching. Vol. 3. Addison-Wesley, Reading, 1998.

[11] MacMahon P.A. Combinatory analysis. University Press, Cambridge, 1915.

[12] Riordan J. An introduction to combinatorial analysis. John Wiley \& Sons, New York, 1958.

[13] Savage C.D., Wilf H.S. Pattern avoidance in compositions and multiset permutations. Adv. Appl. Math. 2006,36 (2), 194-201. doi:10.1016/j.aam.2005.06.003

[14] Stanley R.P. Enumerative Combinatorics. Vol. 1. Cambridge University Press, Cambridge, 1997.

[15] Stanley R.P. Enumerative Combinatorics. Vol. 2. Cambridge University Press, Cambridge, 1999.

[16] Zatorskii R.A. Counting m-submultisets through their secondary specifications. In: Rybnikova K.A. (Ed.) Combinatorial Analysis, 7. MSU, Moscow, 1986. (in Russian)

[17] Zatorskii R.A. On the number of combinations on multisets. Bull. Kyiv Nat. Univ. Ser. Phys. Math. Sci. 2000, 3 , 42-47. (in Ukrainian)

[18] Zatorskii R.A. On an algorithm for calculation of the number of m-permutations on multisets. Mat. Stud. 2002, 17 (2), 215-219. (in Ukrainian)

[19] Zatorskii R.A. Some methods and problems of combinatorial analysis. Lik, Ivano-Frankivsk, 2006. (in Ukrainian)

Махней О.В., Пилипів В.М., Заторський Р.А. m-підмультимножини та т-перестановки елементів мультимножин // Карпатські матем. публ. - 2021. - Т.13, №1. - С. 240-258.

Стаття присвячена двом класичним комбінаторним задачам на мультимножинах, яким у існуючій літературі відведено невиправдано мало місця. А саме: обчисленню числа всіх підмультимножин потужності $m$ довільної мультимножини та числа $m$-перестановок таких мультимножин. Перша задача тісно пов' язана із шириною частково впорядкованої множини всіх підмультимножин мультимножини за включенням $\subseteq$. У статті виділено деякі важливі класи мультимножин. Розглянуто комбінаторні доведення задач про число $m$-підмультимножин та m-перестановок елементів мультимножини. У статті, на основі методу генератрис, будуються економні алгоритми обчислення $m$-підмультимножин та $m$-перестановок елементів мультимножини. У роботі також зроблено короткий огляд результатів, що стосуються цього напрямку досліджень.

Ключові слова і фрази: мультимножина, перестановка. 